\title{
Ovarian Cancer and Primary Peritoneal Carcinoma pT3 TNM Finding v7
}

National Cancer Institute

\section{Source}

National Cancer Institute. Ovarian Cancer and Primary Peritoneal Carcinoma pT3 TNM

Finding v7. NCI Thesaurus. Code C89650.

Ovarian cancer or primary peritoneal carcinoma involving one or both ovaries with microscopically confirmed peritoneal metastasis outside the pelvis. (from AJCC 7th Ed.) 